ACTA UNIVERSITATIS LODZIENSIS

Folia Litteraria Romanica 15, 2020

https://doi.org/10.18778/1505-9065.15.03

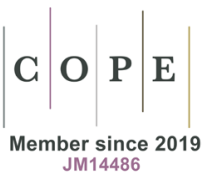

Dariusz Krawczyk

Université de Varsovie

iD ORCID ID : 0000-0002-3407-9592

dariusz.krawczyk@uw.edu.pl

\title{
Le Mépris dans L'Heptaméron de Marguerite de Navarre
}

\begin{abstract}
RÉSUMÉ
Le tableau des grandeurs et des folies, des exploits et des faiblesses humaines dans L'Heptaméron de Marguerite de Navarre ne saurait être complet sans l'évocation du mépris. Ce sentiment n'est jamais sujet principal des nouvelles, mais il se manifeste dans les actions et les jugements des personnages. Cette lecture du recueil met en relief avant tout le mépris comme une des marques de l'orgueil dans sa dimension nobiliaire, courtoise et évangélique.
\end{abstract}

MOTS-CLÉS - Marguerite de Navarre, L'Heptaméron, cuyder, nouvelle française de la renaissance, mépris, orgueil, évangélisme

“Contempt in Marguerite de Navarre's Heptaméron"

\section{SUMMARY}

The picture of wisdom and folly, great deeds and laughable weaknesses in Marguerite de Navarre's Heptaméron, cannot be complete without the evocation of contempt. This feeling is never the main subject of this short stories, but he appears in the moral choices and judgments of the characters. This paper focuses above all on the contempt as one of the marks of pride in its noble, courteous and evangelical dimension.

KEYWORDS - Marguerite de Navarre, L'Heptaméron, French Renaissance Short Stories, contempt, pride, French evangelism

La richesse de l'univers passionnel et moral de L'Heptaméron fait de ce recueil un tableau des vices et des vertus qui baigne dans une troublante ambiguïté. Les histoires se suivent, se répondent par la loi de la continuité ou de l'opposition et des histoires découlent les leçons tirées par les héros de ce qu'on a appelé le « roman virtuel» : les devisants. Et il arrive que, dans ces leçons, ils se contredisent 
et qu'ils reviennent sur les certitudes qui semblaient déjà acquises pour les interroger à nouveau. Les ambiguïtés sont celles de leur société dominée à la fois par les valeurs nobiliaires, chrétiennes et mondaines qui parfois s'accordent et parfois entrent en conflit les unes avec les autres. De ce point de vue la question du mépris, qui sera l'objet de la présente étude, est particulièrement intéressante parce qu'elle permet de cristalliser ces contradictions à travers sa relation avec l'orgueil, vertu nobiliaire par excellence.

Aborder L'Heptaméron par le biais du mépris peut à première vue sembler une tâche ingrate parce qu'il n'est jamais sujet des histoires et n'est pas non plus abordé comme sujet des débats. S'il se manifeste, c'est toujours accessoirement, sur fond de considérations sur l'orgueil, l'honneur, le bien ou la fausse estime de soi. Selon le message véhiculé par les nouvelles, les visées du mépris diffèrent : morales, courtoises, satiriques et par-dessus tout évangéliques. La difficulté réside enfin dans l'impossibilité de distinguer clairement cet affect : dans quelle mesure une critique, une moquerie, une tromperie ou une satire relève-t-elle déjà du mépris? Nous renonçons donc dans le cadre de la présente étude à dresser un panorama du mépris. La difficulté est d'autant plus grande que le relevé des occurrences du mot «mépris» et de ses dérivés dans L'Heptaméron et, de manière non systématique, dans la poésie et le théâtre de la reine de Navarre n'apporte rien de concluant. Ceci ne s'explique pas seulement par l'évolution sémantique (le dictionnaire d'Huguet précise qu'au XVI ${ }^{\mathrm{e}}$ siècle le verbe «mépriser» signifiait commettre une faute, ou se tromper sur le prix ou la valeur ${ }^{1}$ ), parce que même les occurrences des termes qui portent le sème qui nous intéresse (comme «desdain», «contempnement» ou «despris») sont peu nombreuses et ne fournissent pas assez de matière pour développer une interprétation ${ }^{2}$. Visiblement donc les histoires du recueil sont celles de l'amour, de l'honneur, des passions et de vices, mais ne sont pas celles du mépris, ce qui pourrait suggérer que le problème moral de cet affect n'était pas du tout une préoccupation de la reine. Or, la lecture transversale du recueil du point de vue de la place et du sens de l'attitude du mépris et des propos méprisants révèle qu'il est au contraire bien présent et qu'il s'inscrit dans la réflexion morale sur l'homme avant tout dans la perspective évangélique. En effet, aussi bien les jugements des personnages de l'histoire-cadre ainsi que les comportements des héros des histoires laissent apparaître plusieurs facettes du mépris qu'il est possible de diviser grossièrement en deux types : mépris ordinaire et profane et mépris évangélique. Nous adopterons cette division qui organisera nos propos et permettra

1 Voir E. Huguet, Dictionnaire de la langue française du XVI siècle, Classiques Garnier Numérique ; URL : https://www.classiques-garnier.com/numerique-bases/huguet ; consulté le 12.01.2019. Le Dictionnaire du Moyen Français a pourtant noté la présence du terme « mespris » dans le sens proche de l'actuel : «Action méprisable ; honte qui en résulte », Dictionnaire du Moyen Français (1330-1500), dir. Robert Martin; URL : http://www2.atilf.fr/dmf; consulté le 21.11.2020.

2 Voir G. Demerson, G. Proust, Marguerite de Navarre L'Heptaméron. Index, Clermont-Ferrand, Presses Universitaires Blaise Pascal, 2005, p. 137. 
de mettre en évidence le mépris symptomatique de la corruption et celui qui élève et fait la grandeur de l'homme.

Le mépris profane se manifeste le plus facilement à travers l'orgueil nobiliaire, qualité partagée par tous les devisants, étant donné que la petite communauté rassemblée à Sarrance, dans un endroit temporairement coupé du monde, est composée de la haute noblesse de cour. Ces aristocrates prononcent leurs jugements sur le monde et les hommes du haut de leur supériorité sociale, morale et économique. En ce sens, l'orgueil, assimilé à la fierté de soi $^{3}$, est l'une des manifestations de l'honneur et du sentiment de supériorité. C'est donc justement cette idéologie implicite, partagée par la micro-société, qui explique le peu de compassion des devisants envers leurs serviteurs morts ou disparus pendant les inondations : «[ils] eurent une joye inestimable, louans le Createur qui, en se contentant des serviteurs, avoit saulvé les maistres et maistresses $\rangle^{4}$ et Emarsuitte d'ajouter un peu plus loin : «pour perte des serviteurs ne se fault desesperer, car l'on en recouvre assez $)^{5}$. Ces passages trahissent le sentiment du mépris nobiliaire envers ceux qui sont en bas de la société (socialement et surtout moralement) et ce mépris est naturel pour les protagonistes, inculqué dès la naissance avec toutes les autres composantes de l'éthos nobiliaire. Il n'est aucunement présenté comme un problème moral par Marguerite de Navarre, parce que visiblement il n'en était pas un, et nous n'allons pas non plus juger cette attitude, mais ce mépris mérite d'être noté en tant que révélateur de cet univers de valeurs hiérarchique et féodal. Il permet aussi de saisir l'importance de l'orgueil dans les propos de devisants et dans leurs jugements des personnages des histoires.

Un problème moral apparaît pourtant quand le mépris conduit à mettre en doute les valeurs le plus saintes de la société, comme le lien conjugal. Si l'histoire de la Nouvelle 37 est celle de la patience d'une femme trompée par son mari qui finalement parvient à le convaincre de revenir sur la bonne voie, le débat qui suit prend une direction étonnante avec l'intervention de Parlemente. Selon elle, les vertus féminines de patience et de persévérance ne comptent plus quand l'honneur est en jeu :

j'ay oppinion que en mariage [la patience] admene à la fin inimitié. Pource qu'en souffrant injure de son semblable, on est contrainct de s'en separer le plus loing que l'on peult, et de ceste estrangeté là, vient un despris de la faulte du desloyal, et en ce despris peu à peu l'amour diminuë : car, d'autant aime l'on la chose, que l'on en estime la valeur'.

3 Georges Matoré remarque que ce sens positif se manifeste dès le milieu du XVI siècle ; G. Matoré, Le Vocabulaire et la société du XVI siècle, Paris, Presses Universitaires de France, 1988, p. 151.

4 Marguerite de Navarre, L'Heptaméron, éd. N. Cazauran, texte établi par S. Lefèvre (selon l'éd. Gruget), Paris, Gallimard, 2020, coll. Folio, p. 61.

5 Ibid., Prologue, p. 62.

6 Ibid., Nouvelle 37, p. 386. 
Le mépris dont parle Parlamente est la conséquence de la disparition du respect pour le conjoint infidèle. Et ce n'est pas tant l'adultère, mais la position sociale de la personne avec qui il est commis qui importe, parce que son mari délaisse le lit conjugal pour celui de «la plus layde, orde et salle chamberiere qui fut leans» ${ }^{7}$. D'autres commentaires vont dans le même sens : «Je croy, dist Parlamente, que une femme de bien ne seroit poinct si marrie d'estre battue par collere, que d'estre desprisée pour une qui ne la vault pas $»^{8}$. Parlemente et avec elle Longarine font passer les vertus nobiliaires comme l'honneur et l'orgueil avant d'autres valeurs, aussi bien celles qui étaient traditionnellement rattachées à la condition féminine que celles qui devraient caractériser le mariage chrétien. Longarine, jeune veuve, affirme même qu'elle aurait pu tuer son mari par vengeance : «Je l'aimois tant, dist Longarine, que je croy que je l'eusse tué, et me fusse tuée après : car mourir après telle vengeance, m'eust esté chose plus agreable, que vivre loyale avec un desloyal $»^{9}$. Dans ce conflit de valeurs, l'orgueil blessé provoque le mépris (despris), puisque l'amour est lié à la valeur (pris) de l'être aimé. Pour Parlamente et Longarine cette dépréciation est définitive parce que l'amour pour le conjoint ne saurait survivre après une telle injure.

Certes, l'opinion tranchée de Parlamente et de Longarine ne peut être prise pour règle de conduite et le recueil est plein d'exemples de ceux et de celles dont l'amour ne disparaît pas et qui restent fidèles malgré l'infidélité ou la bassesse morale de l'objet de leur amour. Les exemples de vertu (comme Rolandine de la Nouvelle 21, Florinde de la Nouvelle 10 ou la femme de la Nouvelle 38) augmentent la faute de ceux qui s'avilissent et se révèlent indignes de si hauts sentiments. Mais en même temps les propos des deux dames mettent en relief la force destructive du mépris provoqué par l'orgueil blessé quand la femme abandonne la posture d'humilité et de soumission qui devrait en principe être la sienne. Ainsi l'amour est-il subordonné à l'honneur et dans ce sens, la remarque d'Hircan est juste : «vous n'aimez voz mariz que pour vous $\rangle^{10}$.

Car Hircan qui, secondé parfois par Saffredent et Simontault, n'hésite pas à exprimer des idées misogynes et se montre porte-parole du plaisir et de la chair ${ }^{11}$, a le goût de dire les vérités qui dérangent et qui dénoncent la fausseté et l'hypocrisie dans les relations entre les hommes et les femmes. C'est donc lui qui démasque le plus volontiers l'envers de la vertu féminine :

je sçay bien que toujours ung pire diable mect l'autre dehors, et que l'orgueil cherche plus la volupté entre les dames, que ne faict la craincte, ne l'amour de Dieu. [Elles] ont changé

7 Ibid., p. 385.

8 Ibid., p. 386.

${ }^{9}$ Ibid., p. 387.

${ }^{10}$ Ibid.

${ }^{11}$ Comme dans la Nouvelle 4 où il affirme qu'il serait prêt à tuer la vieille servante pour pouvoir violer la dame. 
ce vice en ung plus grand qu'elles tiennent plus honneste. C'est une gloire et cruaulté, par qui elles esperent acquerir nom d'immortalité, et ainsy se gloriffians [...] se font non seullement semblables aux bestes inhumaines et cruelles, mais aux diables, desquelz elles prenent l'orgueil et la malice ${ }^{12}$.

Son mépris pour l'orgueil et l'hypocrisie des femmes, outre de servir potentiellement ses intérêts personnels en facilitant ses conquêtes amoureuses, dévoile une vérité très importante : dans leur vanité elles veulent paraître honorables au prix des souffrances des amants. C'est ainsi qu'il met en évidence une des plus importances failles de cette société où l'honneur masculin et féminin ne sont pas les mêmes ${ }^{13}$ et par conséquent les deux sexes au lieu de tendre vers une harmonie et un respect mutuels se dressent mutuellement des pièges. Son mépris est, certes, provocateur, mais aussi en même temps révélateur.

Mais le mépris manifesté par les devisants envers les héros des histoires n'a pas seulement cette vertu dénonciatrice. Il n'est pas rare en effet que le «petit tribunal des Dix $»^{14}$ s'exprime avec mépris sur ceux et celles qui n'ont pas agi ou parlé comme il fallait. Les membres de ce tribunal ne se posent pas la question de savoir s'ils ont le droit légitime de juger autrui et ils vont parfois très loin dans leurs critiques où se manifeste leur sentiment de supériorité : «C'est grande chose, dist Hircan, qu'en quelque sorte que ce soit, il fault tousjours que les femmes facent mal ${ }^{15}$. Ce n'est que de temps en temps que les propos aussi fermes se trouvent tempérés par d'autres protagonistes en vertu du principe biblique «Ne jugez pas, afin de n'être pas jugés» (Mt 7:1) : «Est-ce pas peché, que de juger son prochain?» dit Nomerfide à la fin de la Nouvelle 14. Longarine ajoute que seul «Dieu, qui juge le cueur $[\ldots]$ donnera sa sentence $»^{16}$. Ces appels restent cependant sans réponse

12 Marguerite de Navarre, L'Heptaméron, op. cit., Nouvelle 26, p. 327-328. Dans le débat qui suit la Nouvelle 42, Saffredent dresse la vision de l'origine de l'honneur féminin : « au commencement que la malice n'estoit trop grande entre les hommes, l'amour y estoit si naifve et forte que nulle dissimullation n'y avoit lieu. [...] Mais, quant l'avarice et le peché vindrent saisir le cueur et l'honneur, ilz en chasserent dehors Dieu et l'amour; et, en leur lieu, prindrent amour d'eulxmesmes, hypocrisie et fiction. Et, voiant les dames nourrir en leur cueur ceste vertu de vraye amour et que le nom d'ypocrysie estoit tant odieux entre les hommes, luy donnerent le surnom d'honneur, tellement que celles qui ne povoient avoir en elles ceste honorable amour, disoient que l'honneur le leur deffendoit, et en ont faict une si cruelle loy, que mesmes celles qui ayment parfaictement, dissimullent, estimant vertu estre vice »; ibid., p. 419. L'honneur féminin est comme le miroir inversé de l'honneur masculin, voir à ce titre I. Maclean, « The Renaissance Notion of Woman », Woman Triumphant : Feminism in French Literature 1610-1652, Oxford, Clarendon Press, 1977, p. 19.

${ }^{13}$ Voir les propos de Parlamente dans cette même Nouvelle 26, Marguerite de Navarre, L'Heptaméron, op. cit., p. 328.

${ }^{14}$ G. Mathieu-Castellani, La Conversation conteuse. Les Nouvelles de Marguerite de Navarre, Paris, Presses Universitaires de France, 1992, p. 16.

${ }^{15}$ Marguerite de Navarre, L'Heptaméron, op. cit., Nouvelle 65, p. 545, et Nouvelle 26, p. 328.

${ }^{16}$ Ibid., Nouvelle 14, p. 198. 
et les protagonistes ne vont pas s'interroger sur la possibilité de sortir de cette autre servitude des passions qu'est le mépris d'autrui dont ils font preuve.

Cette question est d'autant plus importante que certaines nouvelles constituent bien l'illustration de la déchéance morale de certains esprits faibles amorcée par le couple orgueil-mépris. De ce point de vue l'exemple le plus intéressant est donné par la Nouvelle 3 dans laquelle le mépris savamment exploité devient un puissant mobile de séduction. Cette histoire de l'infidélité parallèle de deux couples (d'un côté le roi et la reine de Naples, et de l'autre un gentilhomme et sa belle épouse) montre que dans les relations amoureuses, surtout dans l'innamoramento, il suffit d'un esprit fin en psychologie amoureuse pour transformer l'honneur blessé en étincelle amoureuse. En effet, quand le gentilhomme apprend l'infidélité de son épouse avec le roi de Naples, il ne se décide pas à une confrontation directe, mais à une vengeance secrète pour rendre au roi la pareille. Il choisit de séduire à son tour la reine, ce qu'il réussit non grâce à un long service, mais à son éloquence et à sa capacité à faire appel aux bons mobiles pour faire fléchir la vertu de cette femme honorable. C'est donc en l'abordant quand elle est dans la détresse et blessée dans son amour propre par l'infidélité de son mari qu'il lui parle de l'honneur, du plaisir amoureux dont elle est privée et surtout du mépris de la part de celui qui devait l'honorer :

Et si la pitié de moy, qui meurs pour l'amour de vous, ne vous incite à m'aimer, au moins celle de vous mesme vous y doibt contraindre, qui, estant si parfaicte que vous meritez avoir les cueurs de tous les honnestes hommes du monde, estes desprisée et delaissé de celuy pour qui vous avez dedaigné tous les aultres ${ }^{17}$.

La stratégie de séduction du gentilhomme fait appel à l'éthique nobiliaire et cette vengeance constitue l'exemple de la colère au sens aristotélicien ${ }^{18}$, tout en s'accomplissant dans la sphère privée. Le séducteur-manipulateur vise à éveiller l'indignation, la juste colère contre l'injustice flagrante et le mépris pour celui qui en est la cause. C'est ainsi que la fierté d'être la plus vertueuse et digne des plus hauts honneurs pousse la dame entre les bras du gentilhomme qui accomplit ainsi une double vengeance : la sienne et celle de la reine. La stratégie s'avéra payante, parce que les deux couples se trompent mutuellement, et ce pendant des années. Par un retournement comique, la vertu abaissée du roi trouve son reflet dans la vertu élevée du gentilhomme qui désire le bien du prince auquel il n’aurait jamais dû

${ }^{17}$ Ibid., Nouvelle 3, p. 85-86.

18 « Définissons la colère comme l'appétit accompagné de souffrance de ce qui apparaît comme une vengeance à cause de ce qui apparaît comme un acte de dépréciation atteignant nousmêmes ou nos proches, quand cette dépréciation n'est pas justifiée. Si c'est bien cela la colère, il s'ensuit nécessairement : [...] que toute colère soit suivie d'un certain plaisir inspiré par l'espoir de la vengeance », Aristote, Rhétorique, in Euvres complètes, Paris, Éditions Flammarion, 2014, 1378a30. Voir aussi J. Fillion-Lahille, « La colère chez Aristote », Revue des Études Anciennes, 1970, nº 72-1-2, p. 46-79. 
prétendre. Mais cette histoire joyeuse ne devrait pas cacher un problème moral de la déchéance des deux couples, d'autant plus que dans leur histoire il n'est plus question d'un possible redressement moral et du renoncement à l'erreur, comme c'était le cas de l'épouse fidèle de la Nouvelle 37.

Outre ce mépris qui relève de la misère de l'homme, certaines nouvelles mettent en évidence une manifestation plus rare du mépris, celui qui fait la grandeur humaine. Bien sûr, on reste toujours dans le registre profane et on retrouve les mêmes éléments que dans les nouvelles évoquées précédemment, comme la triade amour-orgueilmépris et le conflit des valeurs, mais certaines nouvelles glorifient le sacrifice de soi des amants vertueux qui méprisent la vie et se retranchent du monde dans la solitude ou la mort. Cette fois-ci cependant, le mépris ne conforte pas l'homme dans son attachement aux valeurs terrestres, mais l'en éloigne, tout en restant dans le registre profane. Il est nécessaire de souligner que les amoureux tirent gloire d'aimer parfaitement, car l'honneur n'est d'habitude pas accompagné d'humilité et cette caractéristique est particulièrement visible dans les débats de Marguerite de Navarre, comme La Coche (1541) ou La Comédie des parfaits amants (1549), ouvrages chronologiquement proches de la rédaction de L'Heptaméron, où il s'agit d'identifier l'amant parfait et de le récompenser par ce titre. La mort d'amour constitue la confirmation in extremis du dévouement, de la fidélité, du service, c'est-à-dire de la perfection dans l'éthique courtoise. C'est ainsi qu'en embrassant sur son lit de mort pour la première et l'unique fois dans sa vie sa bien-aimée, le gentilhomme de la Nouvelle 9 affirme l'extrémité de son sentiment et le peu de prix qu'il attache à la vie :

je sçay que avecq nul autre ne sera si bien traictée, ne tant aymée qu'elle eust esté avecq moy. Le bien que je veois qu'elle perd du meilleur et plus affectionné serviteur et amy qu'elle ayt en ce monde, me faict plus de mal que la perte de ma vie, que pour elle seule je voulois conserver; toutesfois, puis qu'elle ne luy peult de rien servir, ce n'est grand gaing de la perdre ${ }^{19}$.

Sa mort est le triomphe de l'idéal amoureux, tout comme la marque du mépris pour les autres choses terrestres, y compris de sa vie. Ayant porté l'amour pour sa dame à son paroxysme, le gentilhomme était conscient de développer en lui la forme la plus grave de l'amour mélancolique, cette passion brûle littéralement le corps de l'amant et amène inévitablement la mort si une cure adéquate n'est pas administrée à temps ${ }^{20}$. Au moment où le héros meurt, sa valeur éclate au grand jour et rend admirable son dévouement et sa magnificence qui dépasse celle à laquelle pouvait normalement aspirer ce gentilhomme issu de petite noblesse. Ce sommet

${ }^{19}$ Marguerite de Navarre, L'Heptaméron, op. cit., Nouvelle 9, p. 118.

${ }^{20}$ Voir à ce titre par exemple l'analyse de la passion amoureuse et de ses conséquences psychophysiques dans B. Marczuk, Les Maladies de l'âme dans les narrations au féminin à la Renaissance (Hélisenne de Crenne, Jeanne Flore, Marguerite de Navarre), Kraków, Universitas, 2006, p. 46-51. 
de la vertu courtoise est atteint aussi par le Premier Gentilhomme du poème Les Quatre Dames et les Quatre Gentilshommes (vers 1540), preuve que le motif du mépris de la vie d'un vrai amant était encore très présent dans la littérature amoureuse de l'époque. La différence est cependant importante : la vertu du héros de la nouvelle brille sur son lit de mort, tandis que le Premier Gentilhomme a déjà donné cette preuve ultime et s'adresse à sa bien-aimée d'outre-tombe ${ }^{21}$.

La Nouvelle 26 qui raconte l'amour de la femme de Pampelune est en quelque sorte le pendant féminin de ces sacrifices exemplaires masculins. C'est l'histoire de l'humilité admirable d'une femme mariée qui porte l'amour pour le sieur d'Avannes à son paroxysme et qui en meurt heureuse d'avoir parfaitement aimé. Mais c'est aussi l'histoire du mépris de l'orgueil et des plaisirs amoureux, s'ils s'acquièrent au prix de compromis moraux. Bien que de condition inférieure, elle a manifesté une noblesse de cœur et de sentiments bien supérieure à celle de son soupirant, pourtant noble. Sa réponse aux louanges du seigneur d'Avannes, qui voyait en elle la perfection incarnée, est un bon exemple de sa victoire sur l'orgueil et la fausse estime de soi que Marguerite de Navarre appelait le cuyder :

Je sçay très bien que je suis femme, non seullement comme une aultre, mais imparfaicte, que la vertu feroit plus grand acte de me transformer en elle, que de prandre ma forme, sinon quant elle voudroit estre incongneuë en ce monde. Car, soubz tel habit que le myen, ne pourroit la vertu estre recongneuë telle qu'elle est. Si est-ce, Monseigneur, que pour mon imperfection, je ne laisse à vous porter telle affection que doibt et peut faire femme craignant Dieu et son honneur ${ }^{22}$.

Sincèrement humble, la femme refuse l'idée d'être parfaite et met très bien en évidence l'inadéquation entre sa condition et le qualificatif «vertueux» utilisé pour la décrire. Elle s'abaisse, tout comme Françoise de la Nouvelle 42 qui se disait « un ver de terre $»^{23}$. Toutes les deux, elles ressortent victorieuses de l'épreuve parce qu'elles méprisent l'orgueil et font triompher l'humilité et la maîtrise de soi, vertus féminines par excellence, au nom de l'honneur et de l'amour. La vertu de l'héroïne de la Nouvelle 26 éclatera au grand jour sur son lit de mort, même si de sérieux doutes demeurent sur la valeur exemplaire de son comportement ${ }^{24}$.

Dans le registre profane, le mépris s'insinue donc dans les relations amoureuses et apparait sous plusieurs facettes : il masque l'orgueil et la déchéance, peut devenir stimulus à l'amour, dénonce l'hypocrisie et même se manifeste comme une force

${ }^{21}$ Les Quatre Dames et les Quatre Gentilshommes, in Marguerite de Navarre, CEuvres complètes. Tome 5. L'Histoire des Satyres, et Nymphes de Dyane, Les Quatre Dames et les quatre Gentilzhommes, La Coche, éd. A. Gendre, L. Petris, S. de Reyff, Paris, Honoré Champion, 2012, v. 1097-1383.

${ }_{22}$ Marguerite de Navarre, L'Heptaméron, op. cit., Nouvelle 26, p. 320-321.

${ }^{23}$ Ibid., Nouvelle 42, p. 413.

${ }^{24}$ En effet, les devisants sont eux-mêmes partagés au sujet du jugement moral à porter sur l'amour de la bourgeoise, étant donné qu'elle a aimé parfaitement le sieur d'Avannes alors qu'elle était mariée. C'est d'ailleurs l'une des nouvelles les plus commentées par la critique. 
purificatrice qui aide à s'affranchir de la servitude des passions pour atteindre l'idéal de l'éthos courtois.

Pour compléter le tableau du mépris il faut dire que mépris mondain se combine au mépris religieux et plus exactement évangélique, étant donné que le discours amoureux de L'Heptaméron baigne dans l'atmosphère spirituelle d'une rare intensité. Rassemblés dans le monastère de Notre-Dame de Sarrance, les dix devisants partagent leurs journées entre les occupations plaisantes pour le corps et pour l'esprit et, de façon naturelle, leurs réflexions morales tournent autour des problèmes de nature religieuse où sont mis en relief avec force ce que Nicolas Le Cadet a appelé les "lieux communs évangéliques $»^{25}$, comme la faiblesse et l'imperfection humaines, la confiance en Dieu ou la nécessité de la grâce.

On y retrouve bien sûr ce mépris aux vertus purificatrices : le contemptus mundi, mais cette fois-ci sous sa forme du mépris chrétien du monde amplement fondé à la fois sur l'Ancien et le Nouveau Testament, ainsi sur l'enseignement de saint Paul et des pères de l'Église combiné parfois avec la fuga mundi, la fuite du monde ${ }^{26}$. La célèbre correspondance entre Marguerite de Navarre et l'évêque Guillaume Briçonnet des années 1521-1524 aborde cette question et Briçonnet rappelle à son élève la nécessité de mépriser les biens terrestres et les honneurs auxquels le chrétien doit préférer les biens éternels ${ }^{27}$. Ce mépris juste caractériserait selon lui l'attitude du vrai croyant dans le monde : «Ilz bruslent d'embrasser la croix du Seigneur et debonnaire Jesus. Leur vie est contempnement, desolation, delaissement $»^{28}$.

En revanche, Briçonnet n'incite pas Marguerite à fuir le monde, parce qu'à l'attitude d'éloignement et du repli sur soi il préfère une autre qu'il résume dans cette formule très évocatrice dans sa lettre du 31 décembre $1521:$ «Desgantez vous, Madame, et ne laissez le vray et savoureulx feu oisif en voz cœur, yeulx et piedz. Il ne souffit bien sentir, congnoistre et desirer. Il reste executer. L'execution est signe de habitant esperit de Dieu, lequel n'est retardé pour les contradictions $»^{29}$. Le mépris du monde ne se traduira donc pas par une retraite contemplative et l'appel à «executer» prévaudra sur le désir de l'otium religiosum. Toujours est-il que

${ }^{25}$ N. Le Cadet, L'Évangélisme fictionnel. Les Livres rabelaisiens, le Cymbalum Mundi, L'Heptaméron (1532-1552), Paris, Classiques Garnier, 2010, p. 325.

${ }^{26}$ Ce mépris est, entre autres, analysé par Aldo Gennaï dans son étude L'Idéal du repos dans la littérature française du XVIe siècle, Paris, Classiques Garnier, 2011, p. 427-442.

${ }^{27}$ Il en parle dans la lettre 6 datée d'août 1521. Voir aussi la lettre 20 du 22 décembre 1521 : «Aussy doibt tout chrestien fructiffier à Dieu par continuel labeur spirituel et contempnement du monde, qui est le foing, l'asne et le beuf. Nous serons purgéz par contempnement du monde, illuminéz par excercice spirituel et labeur, et parfaictz en fructiffiant par bonnes operations en son amour ", in G. Briçonnet, Marguerite d'Angoulême, Correspondance 1521-1522, éd. Ch. Martineau, M. Veissière, Genève, Droz, t. I, 1975, p. 112.

${ }^{28}$ Lettre 72 du 20 novembre 1523, in G. Briçonnet, Marguerite d'Angoulême, Correspondance 1523-1524, op. cit., t. II, 1979, p. 75.

${ }^{29}$ Lettre 21 du 31 décembre 1521, in G. Briçonnet, Marguerite d'Angoulême, op. cit., t. I, p. 128. 
la fuite du monde ne sera pas étrangère à l'élève de Briçonnet et elle n'oubliera pas de trouver du temps pour ses « solitudes ».

Le mépris fondé sur les convictions religieuses se manifeste dans L'Heptaméron sous la forme du mépris envers les moines. Si tout le recueil possède une dimension satirique et plusieurs groupes sont épinglés (médecins, magistrats, courtisans), les « fascheux cordeliers » ont la particularité d'être le seul personnage collectif à être traité de la sorte ${ }^{30}$. Marguerite ne propose pas une vision sécularisée de l'otium religiosum, comme le fait François Rabelais pour son abbaye de Thélème, mais n'a pas de mots assez durs pour condamner les moines et remplit les journées de son Heptaméron d'histoires de dépravation, d'avarice, de gourmandise et d'impiété de ceux qui étaient censés porter la Parole. Aldo Gennaï remarque à juste titre que sous la plume des humanistes chrétiens érasmisants, le noble otium des moines changeait bien souvent en otiositas, l'oisiveté ${ }^{31}$.

Il arrive ainsi aux devisants de dénoncer la bêtise, la folie, la lâcheté et la déchéance humaines, mais seul le mépris est perceptible dans la manière de raconter les mésaventures et les méfaits des moines. Quand Guebron demande à Oisille de dire quelque chose en leur faveur, elle s'esquive en disant : « Nous avons tant juré, dist Oisille, de dire la verité, que je ne sçaurois soustenir ceste partie $»^{32}$. C'est aussi elle qui demande à Guebron de ne pas ménager les moines : « je le prie qu'il n'espargne poinct les moynes ${ }^{33}$. Si la plus âgée, sage et mesurée du groupe refuse de les défendre, c'est parce qu'ils condensent ce contre quoi s'élèvent les évangéliques ${ }^{34}$. Et leur mépris apparaît comme un acte d'accusation et un appel à une réforme dont l'urgence n'est plus à démontrer et en effet, dans sa vie de reine, Marguerite aura mis beaucoup d'énergie pour réformer les couvents. Il est intéressant de remarquer aussi que, dans sa critique, elle épargne les religieuses qui, à défaut d'être toujours toutes intelligentes et prévoyantes, ne sont jamais aussi corrompues. Contrairement aux idées reçues de l'époque, le désir masculin semble être plus irrépressible que le désir féminin.

${ }^{30}$ Emarsuitte souligne même leur inutilité : " ilz sont si très inutilles, que, s'ilz ne font quelque mal digne de memoire, on n'en parleroit jamais », Nouvelle 48, éd. cit., p. 455. La dimension à la fois évangélique et satirique de L'Heptaméron a déjà fait objet de plusieurs études. Voir entre autres : N. Cazauran, L'Heptaméron de Marguerite de Navarre, Paris, SEDES, 2005, p. 259269 ; J. Perrenoud-Wörner, Rire et sacré : la vision humoristique de la vérité, in L'Heptaméron de Marguerite de Navarre, Genève, Éditions Slatkine, 2008, p. 371-380, et G. Ferguson, « Mal de vivre, mal croire : l'anticléricalisme dans L'Heptaméron de Marguerite de Navarre », Seizième Siècle, 2010, no 6, p. 151-163.

${ }^{31}$ A. Gennaï, op. cit., p. 121.

${ }^{32}$ Marguerite de Navarre, L'Heptaméron, op. cit., Nouvelle 22, p. 285.

${ }^{33}$ Ibid., Prologue de la quatrième journée, p. 347. La Nouvelle 31 narrée par Guebron raconte en effet plusieurs meurtres commis par un franciscain flamand.

${ }^{34}$ À de très rares occasions on trouve quand même une défense timide : « Il y en a de bons, dist Oisille, et ne fault pas que pour les mauvais, ils soient tous jugez : mais les meilleurs sont ceux qui hantent moins les maisons seculieres, et les femmes », ibid., Nouvelle 22, p. 285. 
La critique des moines permet à Marguerite de dénoncer non seulement leur bêtise et leur paillardise, mais aussi le cuyder, c'est-à-dire la présomption humaine qui fait oublier Dieu et mène vers le péché ${ }^{35}$. Sa condamnation remonte à la Bible et au passage du livre de l'Ecclésiastique (Siracide) où il est dit que l'orgueil est le commencement de tous les péchés (initium omnis peccati est superbia; $\mathrm{Si}$ $\left.10: 15^{36}\right)$. C'est sur cette phrase que vont se fonder les réflexions de saint Augustin sur l'orgueil qui provoque le mépris de Dieu : «Et qu'est-ce que 1'orgueil, sinon l'appétit d'une fausse grandeur? C'est une fausse grandeur qui, délaissant Celui à qui l'âme doit demeurer unie comme à son principe, prétend devenir en quelque sorte son principe à soi-même; et cela, quand l'âme se complaît trop en soi » ${ }^{37}$. Pour saint Thomas, tout péché est en quelque sorte un mépris de Dieu, mais l'orgueil a une place à part, parce que le pécheur refuse directement de se soumettre à Dieu ${ }^{38}$. Si saint Benoît a écrit Les douze degrés de l'humilité, saint Bernard y a répondu quelques siècles plus tard par le Traité des degrés de l'humilité et de l'orgueil, en mettant l'accent sur l'humilité qui est le premier degré de la vérité et la connaissance de soi, alors que l'orgueil est au contraire l'ignorance de soi ${ }^{39}$.

Forte de cette tradition théologique, Marguerite réserve au cuyder des gens de l'Église un traitement spécial. Les portraits d'orgueilleux méprisables sont nombreux dans L'Heptaméron, mais l'un d'entre eux se distingue par l'ampleur de sa déchéance physique et morale. Il s'agit du prieur de Saint-Martin qui voulait piéger Marie Héroët dans la célèbre Nouvelle 22. La déchéance du prieur est concomitante à l'inflation de son orgueil :

s'estimant luy-mesme le bien public de toute religion, desira de conserver sa santé mieulx qu'il n'avoit accoustumé. Et, combien que sa reigle portast de jamais ne manger chair, il s'en dispensa luy-mesmes, ce qu'il ne faisoit à nul autre, disant que sur luy estoit tout le faiz de la religion. Parquoy, si bien se festoya, que, d'un moyne bien meigre, il en feit ung bien gras. Et, à ceste mutation de vivre, se feit une mutation de cueur ${ }^{40}$.

${ }^{35}$ Voir à ce titre l'étude de N. Le Cadet, « Le cuyder dans l'œuvre de Marguerite de Navarre », Seizième Siècle, 2011, nº 7, p. 139-157.

${ }^{36}$ Noua Vulgata Bibliorum Sacrorum Editio, Vatican, Libreria Editrice Vaticana, 1986.

${ }^{37}$ Saint Augustin, Euvres complètes, t. 13, La Cité de Dieu, Bar-le-Duc, L. Guérin \& C ${ }^{\text {ie }}$, 1869, liv. XIV, chap. XIII, p. 297.

38 «C'est pourquoi l'éloignement de Dieu et de ses préceptes, qui existe par voie de conséquence dans les autres péchés, appartient par lui-même à l'orgueil dont l'acte est le mépris de la Divinité»; Saint Thomas d'Aquin, La Somme Théologique, trad. C.-J. Drioux, $2^{\mathrm{e}}$ partie de la $2^{\mathrm{e}}$ Partie, Question 162 : De l'orgueil ; URL : http://jesusmarie.free.fr/thomas_d_aquin_somme_theologique.html ; consulté le 30.01.2019.

${ }^{39}$ Le thème du mépris dans la tradition chrétienne est plus amplement développé dans Le Mépris $d u$ monde. La notion du mépris du monde dans la tradition spirituelle occidentale, éd. M. de Certeau, L. Cognet, J. Daniélou et al., Paris, Les Éditions du Cerf, 1965.

${ }^{40}$ Marguerite de Navarre, L'Heptaméron, op. cit., Nouvelle 22, p. 274. 
La fausse estime de soi remplace l'humilité et provoque un renversement dans le comportement moral. Le prieur commence à mépriser tout ce qui constituait son ancienne vie, à savoir la crainte divine, les jeûnes, le refus des jouissances charnelles. Sa laideur morale ressort avec d'autant plus de force qu'il a face à lui sa victime, sœur Marie Héroët, exemplaire par sa foi, son humilité, sa patience et son intransigeance morale. Lui au contraire devient prédateur et sa passion dévorante pour elle tourne en une obsession qui détruit ce qui restait en lui en fait de barrières morales ${ }^{41}$.

Dans ce cas-là, il ne s'agit plus d'un banal mépris des cordeliers bêtes et cupides, certes ignobles, mais somme toute faciles à repérer et relativement peu dangereux. Le péché du cuyder, ce mépris hautain de Dieu caché sous le masque de piété, est, quant à lui, particulièrement dangereux pour la communauté des fidèles et donc doit être puni de manière exemplaire. Il est la marque de l'involution vers la bestialité d'un homme jadis probe, intègre et considéré par les fidèles comme un saint, parce qu'il a oublié que la victoire sur le cuyder n'est jamais définitive et qu'elle nécessite le secours de la grâce : " À dire la verité, dist Parlamente, il est impossible que la victoire de nous-mesmes se face par nous-mesmes, sans ung merveilleux orgueil qui est le vice que chacun doibt le plus craindre, car il s'engendre de la mort et ruyne de toutes les aultres vertuz $»^{42}$. La perspective évangélique du mépris qui sous-tend cette nouvelle éclaire sous un jour nouveau la relation particulière entre le mépris et l'orgueil, l'attachant à la condamnation de l'autosatisfaction et de la fausse idée de grandeur.

L'histoire de l'odieux prieur de Saint-Martin montre bien que, sans être jamais directement abordé en tant que sujet des débats, le mépris dans L'Heptaméron possède une forte valeur didactique qui vise à rappeler que le véritable bonheur réside dans la capacité à rejeter les biens et les tentations de ce monde et à se concentrer sur les valeurs éternelles. C'est le «mespris des choses fortuites» dont parlait Rabelais. Les appels en ce sens ne manquent pas : c'est ainsi que par exemple Dagoucin condamne les désirs immodérés : «L'homme est bien desraisonnable quant il a de quoy se contanter, et veult chercher autre chose. Car j'ay veu souvent, pour cuyder mieulx avoir et ne se contanter de la suffisance, que l'on tombe au pis $\rangle^{43}$. On retrouve aussi dans la bouche d'Oisille le mépris de la libido sciendi et l'exaltation des simples d'esprit : «nul n'est plus ignorant que celluy qui cuide sçavoir $»^{44}$. Ce sont tous des lieux communs évangéliques dont l'importance n'était

${ }^{41}$ « Ainsi s'en alla cest hipocrite à Sainct-Martin, auquel lieu ce meschant feu, qu'il avoit en son cueur, ne cessa de brusler jour et nuict, et de chercher toutes les inventions possibles pour venir à ses fins »; ibid., p. 278.

${ }^{42}$ Ibid., Nouvelle 34, p. 367-368.

${ }^{43}$ Ibid., Nouvelle 8, p. 113.

${ }^{44}$ Ibid., Nouvelle 51, p. 474. Dans la Nouvelle 34, Oisille commente l'Épître aux Romains et ajoute : « ceulx qui ont cuydé estre plus saiges que les aultres hommes, et qui, par une lumiere de raison, sont venuz jusques à congnoistre ung Dieu createur de toutes choses, toutesfois, pour s'attribuer ceste gloire et non à Celluy dont elle venoit, estimans par leur labeur avoir gaingné ce sçavoir, 
pas à démontrer, mais qu'il fallait répéter inlassablement pour qu'ils pénètrent dans les esprits égarés.

Le mépris révèle dans L'Heptaméron bien des facettes : le mépris justifié pour les objets méprisables, le mépris condamnable pour les objets et principes sacrés et cet entre-deux, cette zone difficile à cerner où les principes qui régissent ce monde courtois peuvent être méprisables ou respectables selon les circonstances et les points de vue, comme l'honneur, la fierté, le sens de la dignité. Il peut conduire à des actes louables ou bien au contraire enfoncer encore plus le personnage dans l'erreur et le péché. À défaut d'être le sujet principal des nouvelles, le mépris s'inscrit comme un des symptômes des maux qui rongent l'humanité et qui permet de dévoiler, de « mettre à nu » pour utiliser la formule de Gisèle Mathieu-Castellani ${ }^{45}$, c'està-dire de poser le diagnostic moral du monde de l'hypocrisie, de la dissimulation et de l'égarement spirituel. Des maux auxquels les devisants n'échappent pas non plus, eux qui se sont érigés en tribunal et s'arrogent le droit de juger autrui. Au fil des histoires et des débats ils se révèlent eux-mêmes faibles et sujets aux passions au même titre que ceux qu'ils critiquent et même parfois méprisent. En apparence, il n'est possible de déceler dans leurs propos aucune évolution au fil des journées, comme si les histoires qu'ils commentent n'avaient aucune influence sur eux. Mais au fond, la retraite involontaire qui s'apparente à la vie en communauté religieuse à Sarrance montre ses fruits, parce que, tout en portant des jugements différents sur les choses profanes, les protagonistes non seulement s'accordent sur les points de la foi, mais vivent ensemble une progression spirituelle. Les prologues et les épilogues de chaque journée en témoignent et dessinent subtilement cette évolution qui intervient grâce à la direction spirituelle d'Oisille, mais aussi à cette réflexion sur la grandeur et la petitesse de l'homme à laquelle ils s'adonnent. Comme si finalement ceux qui s'étaient juré de partager leurs journées à parts égales entre le repos spirituel et un «passe temps et exercice corporel $»^{46}$ en arrivaient progressivement à mépriser ce qui est bas et contingent pour se concentrer sur l'essentiel.

ont esté faictz non seulement plus ignorans et desraisonnables que les aultres hommes, mais que les bestes brutes ? Car, ayans erré en leurs esperitz, s'attribuans ce que à Dieu seul appartient, ont monstré leurs erreurs par le desordre de leurs corps, oblians et pervertissans l'ordre de leur sexe », p. 368. Sur le thème de l'ignorance voir N. Cazauran, Variétés pour Marguerite de Navarre. 1978-2004. Autour de L'Heptaméron, Paris, Honoré Champion, 2005, « Marguerite de Navarre et le vocabulaire de l'ignorance », p. 31-56.

${ }^{45}$ « Le projet global de L'Heptaméron est en effet de découvrir et de mettre à nu ce que femmes et hommes dissimulent par leurs feintes et leurs artifices, consciemment ou inconsciemment : toute l'œuvre, [...] se soutient de la dialectique de la couverture et de l'ouverture »; G. MathieuCastellani, op. cit., p. 233.

${ }^{46}$ Marguerite de Navarre, L'Heptaméron, op. cit., Prologue, p. 64. 


\section{Bibliographie}

Aristote, Euvres complètes, dir. Pierre Pellegrin, Paris, Éditions Flammarion, 2014

Augustin, saint, Euvres complètes, t. 13, La Cité de Dieu, trad. Émile Saisset, Bar-le-Duc, L. Guérin $\& \mathrm{C}^{\mathrm{ie}}, 1869$

Cazauran, Nicole, «Marguerite de Navarre et le vocabulaire de l'ignorance», Variétés pour Marguerite de Navarre. 1978-2004. Autour de L'Heptaméron, Paris, Honoré Champion, 2005, p. 31-56

Cazauran, Nicole, L'Heptaméron de Marguerite de Navarre, Paris, SEDES, 2005

Demerson, Guy; Proust, Gilles, Marguerite de Navarre L'Heptaméron. Index, Clermont-Ferrand, Presses Universitaires Blaise Pascal, 2005

Dictionnaire du Moyen Français (1330-1500), dir. Robert Martin ; URL : http://www2.atilf.fr/dmf ; consulté le 21.11.2020

Ferguson, Gary, «Mal de vivre, mal croire : l'anticléricalisme dans L'Heptaméron de Marguerite de Navarre», Seizième Siècle, 2010, nº 6, p. 151-163 https://doi.org/10.3406/xvi.2010.980

Fillion-Lahille, Janine, «La colère chez Aristote», Revue des Études Anciennes, 1970, n 72-1-2, p. 46-79 https://doi.org/10.3406/rea.1970.3862

Gennaï, Aldo, L'Idéal du repos dans la littérature française du XVIe siècle, Paris, Classiques Garnier, 2011

Guillaume Briçonnet, Marguerite d'Angoulême, Correspondance, t. 1, 1521-1522, t. 2, 1523-1524, éd. Christine Martineau, Michel Veissière, Droz, Genève, 1975 et 1979

Huguet, Edmond, Dictionnaire de la langue française du XVI siècle, Classiques Garnier Numérique; URL : https://www.classiques-garnier.com/numerique-bases/huguet; consulté le 20.01.2019

Le Cadet, Nicolas, «Le cuyder dans l'œuvre de Marguerite de Navarre», Seizième Siècle, 2011, ${ }^{\circ}$ 7, p. 139-157 https://doi.org/10.3406/xvi.2011.1026

Le Cadet, Nicolas, L'Évangélisme fictionnel. Les Livres rabelaisiens, le Cymbalum Mundi, L'Heptaméron (1532-1552), Paris, Classiques Garnier, 2010

Le Mépris du monde. La notion du mépris du monde dans la tradition spirituelle occidentale, éd. Michel de Certeau, Louis Cognet, Jean Daniélou et al., Paris, Les Éditions du Cerf, 1965

Maclean, Ian, The Renaissance Notion of Woman, Woman Triumphant: Feminism in French Literature, 1610-1652, Oxford, Clarendon Press, 1977, p. 19

Marczuk-Szwed, Barbara, Les Maladies de l'âme dans les narrations au féminin à la Renaissance (Hélisenne de Crenne, Jeanne Flore, Marguerite de Navarre), Kraków, Universitas, 2006

Marguerite de Navarre, L'Heptaméron, éd. Nicole Cazauran, texte établi par Sylvie. Lefèvre, Paris, Gallimard, 2020, coll. Folio

Marguerite de Navarre, Euvres complètes. Tome 5, éd. André Gendre, Loris Petris, Simone de Reyff, Paris, Honoré Champion, 2012

Mathieu-Castellani, Gisèle, La Conversation conteuse. Les Nouvelles de Marguerite de Navarre, Paris, Presses Universitaires de France, 1992 https://doi.org/10.3917/puf.mathi.1992.01

Matoré, Georges, Le Vocabulaire et la société du XVI siècle, Paris, Presses Universitaires de France, 1988 https://doi.org/10.3917/puf.mator.1988.01

Noua Vulgata Bibliorum Sacrorum Editio, Vatican, Libreria Editrice Vaticana, 1986

Perrenoud-Wörner, Judith, Rire et sacré : la vision humoristique de la vérité dans L'Heptaméron de Marguerite de Navarre, Genève, Éditions Slatkine, 2008

Thomas d'Aquin, saint, La Somme Théologique, trad. Claude-Joseph Drioux, Paris, Belin, 1852 ; URL : http://jesusmarie.free.fr/thomas_d_aquin_somme_theologique.html; consulté le 30.01.2019 
Dariusz Krawczyk est maître de conférences à l'Université de Varsovie, spécialiste de la poésie spirituelle de la Renaissance, notamment de Marguerite de Navarre et de son cercle évangélique.

(c) creative

(C) by the author, licensee Łódź University - Łódź University Press,

Łódź, Poland. This article is an open access article distributed under the terms and conditions of the Creative Commons Attribution license CC-BY-NC-ND 4.0 (https://creativecommons.org/licenses/by-nc-nd/4.0/)

Received: 2019-01-14; Accepted: 2020-12-15 